\title{
COMMISSION 12: SOLAR RADIATION AND STRUCTURE
} (RAYONNEMENT ET STRUCTURE SOLAIRES)

\author{
PRESIDENT: S.K. Solanki \\ VICE-PRESIDENT: T. Bogdan
}

ORGANIZING COMMITTEE: G. Cauzzi, J. Christensen-Daalsgard,

L.E. Cram, D. Dravins, P.V. Foukal, P. Heinzel, A. Kosovichev, J.T. Mariska, M.G. Rovira, P. Venkatakrishnan \& J. Wang

\section{Introduction}

Commission 12 covers research on the internal structure and dynamics of the Sun, the "quiet" solar atmosphere, solar radiation (including irradiance) and its variability. There is considerable unavoidable and productive overlap with the other Commissions in Division II. Much of it is caused by the fact that the solar magnetic field, which causes active phenomena such as flares, coronal mass ejections and the associated high energy radiation and particle acceleration and also plays an important role in accelerating the solar wind or modulating the cosmic ray flux in the heliosphere, is produced by dynamo action involving the Sun's differential rotation and convective motions, topics that fall under the jurisdiction of Commission 12. In addition, the same magnetic field also produces the more gentle variation of the solar output (irradiance $+p$-mode frequency) over 11 years, the quiet network, subtle changes in solar convection, etc., phenomena covered by Commission 12 . However, the Commissions often consider similar phenomena from very different viewpoints, leading to a deeper insight.

In the last three years significant progress was achieved in almost all of the many topics covered by Commission 12, which cannot be covered in this brief report. A few topics have therefore been picked out, for which brief overviews are given in the following. The selection does not follow any deeper principle, but has rather been guided by the specializations of members of the Organizing Committee of Commission 12 and the idiosyncracies of the Chairman. In particular, it must be stressed that topics not covered in this report have also undergone considerable progress. Examples are solar irradiance variability or solar dynamo theory, in both of which fields considerable advances, both qualitative and quantitative, have been achieved. Finally, solar physics is currently so dynamic that it is impossible to cover all the significant progress in the field even of the topics explicitly reviewed in this report.

\section{Magnetoconvection}

\section{T. Bogdan}

The interaction between magnetic fields and convection is interesting both because of its astrophysical importance and because the nonlinear Lorentz force induces an especially bewildering variety of complex dynamic behaviour. Recent progress has been rapid and wide-ranging. It has been driven principally by a further increase in computing resources and capabilities coupled with additional remarkable high-resolution observations of solar surface magnetic fields. The application of powerful analytic techniques borrowed from the mathematical discipline of nonlinear dynamics functions as the amalgam that binds simulation and observation into a sound physical foundation. 
Advances in computing power have at last made it possible to develop models of turbulent magnetoconvection that can be directly related to these observations. The key features of flux emergence and annihilation, as observed by the MDI experiment on SOHO, or the G-band movies registered by the Swedish telescope on La Palma, are reproduced in the kinematic calculations. Three-dimensional numerical experiments reveal the underlying dynamical processes. For example, we are now confident that turbulent convection is itself able to act as a small-scale dynamo, regenerating the disordered surface fields that are largely unaware of the solar rotation.

The current numerical experiments on three-dimensional magnetoconvection in a stratified compressible layer reveal a range of different patterns. Both the strength of the imposed magnetic field and the aspect ratio of the simulations act in concert to dictate the outcomes. As the influence of the imposed field is decreased there is a transition from small-scale plumes, in the magnetically dominated regime, to large-scale vigorous plumes when the field is dominated by the motion. At sufficiently large aspect ratios, the intermediate regime is characterized by magnetic flux separation. In other words, there are almost field-free regions, with clusters of vigorous plumes, surrounded by regions where the Lorentz force is strong enough to control the dynamics. This is a well known property of steady laminar convection, but what is exciting about this finding is that similar behaviour obtains in fully turbulent fluids.

Another crucial "computational" discovery is the tendency for magnetic flux to be preferentially transported downward out of a turbulent convecting region and subsequently stored in a stably stratified region which lies below. This "magnetic pumping" process is a necessary ingredient for the efficient operation of the large-scale (i.e., rotationallyinfluenced) solar interface dynamo. It may be responsible for the cyclic transport of magnetic flux from the solar convection zone to the stable overshoot region. A rather unexpected but still quite preliminary finding suggests that the process might even be capable of storing some of these fields within the interior of the convection zone. A series of simulations incorporating a solar-like latitudinal shear show that a toroidal field of several tens of kilo-Gauss may be effectively held down by this turbulent flux pumping mechanism.

A much-anticipated new development is the establishment of critical points of contact between the numerical simulations and the traditional mean-field-electrodynamics approach to magnetoconvection. Statistical analyses of the simulations are now beginning to reveal the curious manner in which the Lorentz force acts to quench the turbulent alpha and eta effects. In some instances, one has been able to uncover certain physical processes which lead to a residual dissipation of magnetic field or add to the magnetic helicity. The convergence of these two philosophies and their continued exchange of ideas and puzzles with the next generation of high-resolution solar observations, like the Advanced Technology Solar Telescope and the Solar-B instrument complex, give every assurance that the pace of our progress in understanding magnetoconvection will continue to accelerate far into the 21st century.

\section{References}

Dorch, S.B.F., \& Nordlund, A. 2001, A\&A, 365(3), 562-570.

Hurlburt, N.E., Matthews, P.C., \& Rucklidge, A.M. 2000, Solar Phys., 192(1-2), 109-118.

Ossendrijver, M., Stix, M., \& Brandenburg, A. 2001, A\&A, 376(2), 713-726.

Tobias, S.M., Brummell, N.H., Clune, T.L., \& Toomre, J. 2001, ApJ, 549(2), 1183-1203.

Weiss, N. 2001, Astron. Geophys., 42(3), 10-17. 


\section{Global Helioseismology}

\section{J. Christensen-Dalsgaard}

The preceeding three-year period has witnessed the steady accumulation of high-quality helioseismic data, from the GONG and BiSON networks as well as from the instruments on the SOHO spacecraft. The availability of such nearly continuous data over an extended period, in the case of spatially resolved observations since the last solar minimum and for disc-averaged data for much longer, has allowed investigations of subtle time-varying phenomena, as discussed below. The helioseismic analyses are based on $p$ - and $f$-modes of frequencies as low as $1000 \mu \mathrm{Hz}$. Although the ongoing search for g-modes has yielded potential identifications (e.g. Gabriel et al. 2002), the detectability of g-modes is still in some doubt, the observational upper limit in velocity observations being below $1 \mathrm{~cm} \mathrm{~s}$ (Appourchaux et al. 2000).

The spherically symmetric component of solar internal structure, particularly the sound speed, can be inferred in most of the Sun by inversion of the average multiplet frequencies. The results are generally in reasonable agreement with the so-called 'standard' solar models, but not with models where a substantial reduction in the solar neutrino flux has been attempted (e.g. Bahcall et al. 2001). Correspondingly, models adjusted to match the helioseismically determined structure yield neutrino fluxes close to those of standard models (Turck-Chièze et al. 2001). This strong suggestion for a non-astrophysical solution to the solar neutrino problem was dramatically confirmed by the direct detection of neutrino flavour transitions from the Sudbury Neutrino Observatory (Ahmad et al. 2001, 2002), yielding neutrino production rates in the solar core which are consistent with the standard models. There remain, however, significant, if modest, differences between the helioseismic inferences and the models, most noticeable a region just below the convection zone where the inferred sound speed exceeds that of the models; this difference can be reduced by introducing weak mixing in this region (e.g. Brun et al. 1999). The sensitivity of the solar sound speed to the thermodynamic state of solar matter allows detailed tests of even subtle details in the equation of state (Basu et al. 1999; Di Mauro et al. 2002). An interesting example is the relativistic effects on electrons in the solar core, erroneously omitted in earlier versions of the thermodynamic tables; including them brings into perfect agreement the solar age as determined from fitting the observed frequencies with the age inferred from meteorites (Bonanno et al. 2002).

Solar internal rotation has been inferred in much of the Sun, as a function of distance $r$ to the centre and latitude. Rotation of the deep interior remains difficult to determine, since only a few p modes have even modest sensitivity to the core rotation. Careful analyses of BiSON data (Chaplin et al. 1999, 2001) were consistent with solid body rotation of the radiative interior, although with substantial uncertainties below $0.25 R_{\odot}, R_{\odot}$ being the solar surface radius. Little significant variation with latitude is found in the radiative interior, whereas rotation in the convection zone approximately shares the latitude variation observed on the surface. The interface between these two regions, known as the tachocline, shows strong radial rotational shear. Properties of the tachocline, which are likely of substantial significance for the generation of the large scale solar magnetic field and the solar cycle, were determined by, for example, Charbonneau et al. (1999).

Analyses of the solar internal rotation over the past 6-7 years have revealed significant time variations. Bands of slightly slower and faster rotation are found to move towards the equator on the time scale of the solar cycle. These are very similar to the so-called torsional oscillations first observed on the solar surface by Howard \& LaBonte (1980); however the helioseismic results show that they extend at least over the outer $10 \%$ of the solar radius (Howe et al. 2000; Antia \& Basu 2000, 2001), with some variations being visible throughout the convection zone (Vorontsov et al. 2002). Fairly substantial temporal variations in high latitude rotation were found by Schou (1999) and Antia \& Basu (2001). Surprisingly, evidence for oscillatory variations, with a period of $1.3 \mathrm{yr}$, in equatorial rotation at and just below the tachocline was found by Howe et al. (2000), although the significance of similar 
results was doubted by Basu \& Antia (2001). Recent data have shown that these variations have died out since the middle of 2000. Although this makes more difficult a definite confirmation of their reality, such behaviour is hardly surprising in a complex dynamical system like the solar interior; it is possible, for example, that the 1.3-yr oscillations are associated with the rising part of the solar cycle. Needless to say it is essential that continual helioseismic observations, with sufficient sensitivity to study such subtle effects, be carried out over at least one full solar cycle, and ideally for much longer.

An extensive review of helioseismology, covering the period up to the early part of 2002, was presented by Christensen-Dalsgaard (2002).

\section{References}

Ahmad, Q. R., Allen, R. C., Andersen, T. C., et al., 2001, Phys. Rev. Lett., 87, 071301(1-6). Ahmad, Q. R., Allen, R. C., Andersen, T. C., et al., 2002, Phys. Rev. Lett., 89, 011301(1-6).

Antia, H. M. \& Basu, S., 2000, ApJ, 541, 442-448.

Antia, H. M. \& Basu, S., 2001, ApJ, 559, L67-L70.

Appourchaux, T., Fröhlich, C., Andersen, B., Berthomieu, G., Chaplin, W. J., Elsworth, Y., Finsterle, W., Gough, D. O., Isaak, G. R., Kosovichev, A. G., Provost, J., Scherrer, P. H., Sekii, T. \& Toutain, T., 2000, ApJ, 538, 401-414.

Bahcall, J. N., Pinsonneault, M. H. \& Basu, S., 2001, ApJ, 555, 990-1012.

Basu, S. \& Antia, H. M., 2001, MNRAS, 324, 498-508.

Basu, S., Däppen, W. \& Nayfonov, A., 1999, ApJ, 518, 985-993.

Bonanno, A., Schlattl, H. \& Paternò, L., 2002, A\&A, 390, 1115-1118.

Brun, A. S., Turck-Chièze, S. \& Zahn, J. P., 1999, ApJ, 525, 1032-1041. (Erratum: ApJ, $536,1005)$.

Chaplin, W. J., Christensen-Dalsgaard, J., Elsworth, Y., Howe, R., Isaak, G. R., Larsen, R. M., New, R., Schou, J., Thompson, M. J. \& Tomczyk, S., 1999, MNRAS, 308, 405-414.

Chaplin, W. J., Elsworth, Y., Isaak, G. R., Marchenkov, K. I., Miller, B. A. \& New, R., 2001, MNRAS, 327, 1127-1136.

Charbonneau, P., Christensen-Dalsgaard, J., Henning, R., Larsen, R. M., Schou, J., Thompson, M. J. \& Tomczyk, S., 1999, ApJ, 527, 445-460.

Christensen-Dalsgaard, J., 2002, Rev. Mod. Phys., Oct. 2002, in press [astro-ph/0207403].

Di Mauro, M. P., Christensen-Dalsgaard, J., Rabello-Soares, M. C. \& Basu, S., 2002, A\&A, 384, 666-677.

Gabriel, A. H., Baudin, F., Boumier, P., García, R. A., Turck-Chièze, S., Appourchaux, T., Bertello, L., Berthomieu, G., Charra, J., Gough, D. O., Pallé, P. L., Provost, J., Renaud, C., Robillot, J.-M., Roca Cortés, T., Thiery, S. \& Ulrich, R. K., 2002,. A\&A, 390, 1119-1131.

Howard, R. \& LaBonte, B. J., 1980, ApJ, 239, L33-L36.

Howe, R., Christensen-Dalsgaard, J., Hill, F., Komm, R. W., Larsen, R. M., Schou, J., Thompson, M. J. \& Toomre, J., 2000, ApJ, 533, L163-L166.

Howe, R., Christensen-Dalsgaard, J., Hill, F., Komm, R. W., Larsen, R. M., Schou, J., Thompson, M. J. \& Toomre, J., 2000, Sci, 287, 2456-2460.

Schou, J., 1999, ApJ, 523, L181-L184.

Turck-Chièze, S., Couvidat, S., Kosovichev, A. G., Gabriel, A. H., Berthomieu, G., Brun, A. S., Christensen-Dalsgaard, J., García, R. A., Gough, D. O., Provost, J., Roca-Cortes, T., Roxburgh, I. W. \& Ulrich, R. K., 2001, ApJ, 555, L69-L73.

Vorontsov, S. V., Christensen-Dalsgaard, J., Schou, J., Strakhov, V. N. \& Thompson, M. J., 2002, Sci, 296, 101-103. 


\section{The Solar Chromosphere}

\section{P. Heinzel and R.J. Rutten}

Here we review the progress in understanding the structure and dynamics of the quiet solar chromosphere. The extensive work on oscillations in the chromosphere has been reviewed by Carlsson (1999), Deubner \& Steffens (1999), Heinzel (2000), and Rutten (1999, 2001). After the internetwork "three-minute" oscillations were identified as weak acoustic shocks, the issues under debate are the degree of correlation between internetwork grain occurrence and enhanced internetwork field, the presence of identifiable pistons causing enhanced acoustic flux higher up in the atmosphere, the relation between such pistons and observed velocity-intensity phase-difference patterns in the photosphere (with additional interest concerning $p$-mode asymmetries), the amount of wave reflection in the upper chromosphere, the penetration height of the shocks into the higher chromosphere (as evidenced by ultraviolet spectrometry with HRTS and SUMER), and the shock contribution to chromospheric heating and to the basal flux observed from cool stars. References to all these issues are given in Krijger et al. (2001), recent papers on these topics include Sivaraman et al. (2000), McIntosh \& Judge (2001), McIntosh et al. (2001), Rosenthal et al. (2002) and Hoekzema et al. (2002). Internetwork chromospheric oscillations were investigated using SOHO/SUMER and TRACE observations (Judge et al. 2001). The observed photospheric and chromospheric oscillations are strongly coupled for frequencies between 2 and $8 \mathrm{mHz}$. Phase coherences decrease with increasing height. The middle chromosphere oscillates in several $\mathrm{Mm}$ diameter coherent patches with power predominantly in the $5-7 \mathrm{mHz}$ range. The chromosphere oscillates primarily in response to forcing by the $p$-modes, large-scale (several $\mathrm{Mm}$ across) waves are often strongly influenced by magnetic effects (e.g. canopies). Coordinated space (SOHO/SUMER and TRACE) and ground based (VTT, GCT) observations were reported by Curdt et al. (1999) who analysed hydrogen Lyman lines and continuum oscillations, together with Ca II grains. They show clear evidence of oscillations in the L $\beta$ line, both in intensity and Doppler shifts (asymmetries). Krijger et al. (2002) have further correlated well aligned SUMER, TRACE and VTT $x-t$ oscillatory patterns.

Network oscillations are often invoked to supply chromospheric or coronal heating, but there is no firm identification of any definite mode yet. For references see Sivaraman et al. (2000) and Krijger et al. (2001). Banerjee et al. (2001) used SOHO/CDS and SUMER data to study the nature of network oscillations which are considered to be due to waves produced in short bursts with coherence times of about $10-20 \mathrm{~min}$. The observed $2-4 \mathrm{mHz}$ network oscillations can be interpreted in terms of kink and sausage waves propagating upwards along the magnetic flux tubes. Long-period waves (4-15 min) were found in the central portions of network bright points, indicating their magnetoacoustic or magnetogravity nature (McAteer et al. 2002).

Recent theoretical work on chromospheric modelling includes new radiation hydrodynamics 3D simulations (Wedemeyer et al. 2000) extending from the upper convection zone to the middle chromosphere. Carlsson \& Stein (2002) have investigated the dynamic hydrogen ionization with the conclusion that the ionization of hydrogen in the chromosphere is dominated by collisional excitation in the $L \alpha$ transition followed by photoionization by the Balmer continuum. Finally, Uitenbroek (2002) has demonstrated the importance of partial redistribution for radiation losses in the $\mathrm{Ca}$ II $\mathrm{K}$ line.

Chromospheric and transition-region models have been further refined by Fontenla et al. (2002), who considered the hydrogen and helium mass flows with ambipolar diffusion. The semiempirical chromospheric model exhibits a temperature rise, in contrast to mean models of Carlsson \& Stein which are close to radiation equilibrium (up to $1.8 \mathrm{Mm}$ ). The latter models were criticized by Kalkofen et al. (1999) and Kalkofen (2001) who argue that the observed UV emission lines require an increasing mean temperature. Hundreds of such lines have been detected by SOHO/SUMER and identified in the new SUMER spectral atlas (Curdt et al. 2001). 


\section{References}

Banerjee, D., O'Shea, E., Doyle, J. G., \& Goossens, M. 2001, A\&A, 371, 1137.

Carlsson, M. 1999, in: Magnetic Fields and Solar Processes, Proc. 9th European Meeting on Solar Physics, A. Wilson (ed.), ESA SP-448, ESTEC, Noordwijk, 183.

Carlsson, M., \& Stein, R. F. 2002, ApJ, 572, 626.

Curdt, W., Heinzel, P., Schmidt, W., Tarbell, T. D., von Uexküll, M., \& Wilken, V. 1999, in: Magnetic Fields and Solar Processes, Proc. 9th European Meeting on Solar Physics, A. Wilson (ed.), ESA SP-448, ESTEC, Noordwijk, 177.

Curdt, W., Brekke, P., Feldman, U., Wilhelm, K., Dwivedi, B. N., Schühle, U., \& Lemaire, P. 2001, A\&A, 375, 591.

Deubner, F.-L. \& Steffens, S. 1999, in: Magnetic Fields and Solar Processes, Proc. 9th European Meeting on Solar Physics, A. Wilson (ed.), ESA SP-448, ESTEC, Noordwijk, 149.

Fontenla, J. M., Avrett, E. H., \& Loeser, R. 2002, ApJ, 572, 636.

Heinzel, P. 2000, in: Advances in Solar Research at Eclipses from Ground and from Space, J.-P. Zahn and M. Stavinschi (eds.), NATO ASI, Kluwer, Dordrecht, 201.

Hoekzema, N. M., Rimmele, T. R., \& Rutten, R. J. 2002, A\&A, in press.

Judge, P. G., Tarbell, T. D., \& Klaus, W. 2001, ApJ, 554, 424.

Kalkofen, W. 2001, ApJ, 557, 376.

Kalkofen, W., Ulmschneider, P., \& Avrett, E. H. 1999, ApJ, 521, L141.

Krijger, J. M., Rutten, R. J., Lites, B. W., et al. 2001, A\&A, 379, 1052.

Krijger, J. M., Heinzel, P., Curdt, W., \& Schmidt, W. 2002, A\&A, submitted.

McAteer, R. T. J., Gallagher, P. T., \& Williams, D. R. 2002, ApJ, 567 L165.

McIntosh, S. W., \& Judge, P. G. 2001, ApJ, 561, 420.

McIntosh, S. W., Bogdan, T. J., Cally, P. S., et al. 2001, ApJ, 548, L237.

Rosenthal, C. S., Bogdan, T. J., Carlsson, M., et al. 2002, ApJ, 564, 508.

Rutten, R. J. 1999, in: Magnetic Fields and Oscillations, Proc. Third Adv. in Solar Physics Euroconf., B. Schmieder, A. Hofmann, \& J. Staude (eds.), ASP Conf. Ser., vol. 184, 181.

Rutten, R. J. 2001, in: Cool Stars, Stellar Systems and the Suil, Proc. 11th Cambridge Workshop, R. J. García Lopez, R. Rebolo, \& M. R. Zapatero Osorio (eds.), ASP Conf. Ser., vol. 223, 117.

Sivaraman, K. R., Gupta, S. S., Livingston, W. C., et al. 2000, A\&A, 363, 279.

Uitenbroek, H. 2002, ApJ, 565, 1312.

Wedemeyer, S., Freytag, B., Steffen, M., \& Holweger, H. 2000, Astron. Gesellschaft Abstract Ser., vol. 17.

\section{Local Helioseismology}

\section{A. Kosovichev}

Local helioseismology is a new field of solar physics that studies the three-dimensional structure and dynamics of the solar interior by analyzing local properties of solar oscillations and waves, such as travel times, phase shifts, frequency and amplitude variations. During the past 3 years, 1999-2002, significant progress has been made in developing observational, theoretical and data analysis methods, as well as in the diagnostics of solar phenomena. Local helioseismology is based on a set of observational and data analysis tools, such as 
ring-diagram analysis (Hernandez et al. 2000); time-distance helioseismology (Duvall \& Kosovichev 2001), acoustic holography (Lindsey \& Braun 2000a) and acoustic imaging (Chou 2000).

The major achievements include results for the subsurface structure and dynamics of active regions and sunspots, large-scale flows in the convection zone and their variations with the solar cycle.

\subsection{Sunspots and Active Regions}

The first maps of the sound-speed distribution beneath sunspots have been obtained by the method of time-distance helioseismology. These maps have shown the subsurface structures of relatively low sound speed, which correspond to the cool areas of sunspots and extend up to $4 \mathrm{Mm}$ below the photosphere. In the deeper sunspot structures, the sound-speed is higher than in the surrounding plasma. The depth of these structures is at least $30 \mathrm{Mm}$ which is about the current resolution limit. The higher sound speed in these structures can be due to higher temperature caused by heat accumulation or strong magnetic field (Kosovichev et al. 2000; Jensen et al. 2001).

The strong converging downflows of plasma beneath the sunspots have been discovered by analyzing travel time delays of acoustic waves (p-mode) propagating under the spots. These plasma flows originate near the surface and are 5-6 Mm deep. In deeper layers the flow pattern is reversed; it is dominated by diverging upflows. These results are consistent with Parker's cluster model of sunspots, and also with recent numerical simulations. In addition, strong horizontal vortex motions are found beneath a twisting sunspot. Close to the surface the sunspot flows have been studied by the time-distance analysis of surface gravity waves ( $f$ mode). The results have provided measurements for the near-surface outflows corresponding to the Evershed and moat flows. By comparing the $p$ - and $f$-mode results it has been concluded that the Evershed flow is very shallow, perhaps, only a few hundred kilometres deep (Zhao et al. 2001; Hurlburt \& Rucklidge 2000; Gizon et al. 2000; Kosovichev et al. 2002a).

Studies of emerging active regions by the time-distance method have revealed that the magnetic flux tubes emerge very rapidly, propagating in the upper convection zone with a speed of about $1.3 \mathrm{~km} / \mathrm{s}$; this is faster than predicted by theory. These studies also have provided evidence that solar active regions are formed as a result of emergence of numerous magnetic structures in the same areas, and are not a result of emergence of a large Omega-type loop (Kosovichev et al. 2000; Kosovichev et al. 2002b).

The first images of the solar active regions on the far side of the Sun have been obtained by acoustic holography. These observations are important for long-term space weather forecasts, providing the information about the evolution of active regions on the far side and detecting the emergence of new large regions. The daily far-side images are now produced routinely from the SOHO/MDI data and are posted on the SOI-Stanford web site (Lindsey \& Braun 2000b; Braun \& Lindsey 2001).

\subsection{Large-Scale Flows in the Convection Zone}

Variations of meridional circulation of the Sun with the solar cycle have been studied by the time-distance and ring-diagram methods. The results show that the meridional flows in the upper convection zone vary significantly with the cycle, forming additional circulation cells centered at the active latitudes as solar activity increases. The meridional flows become asymmetrical and may even change the direction from polarward to equatorward. These results are of prime importance for solar dynamo theories (Giles 1999; Chou \& Dai 2001; Haber et al. 2002).

Recent analyses of large-scale subsurface flow patterns have confirmed earlier results about the existence of converging flows around active regions. The East-West component of these flows averaged over longitude reproduces the pattern of 'torsional oscillations'. These 
results have also shown the existence of evolving large-scale shear flows in active regions. The studies of the interaction between the subsurface flows and solar activity have led to a concept of Solar Subsurface Weather (Gizon et al. 2001; Basu \& Antia 2000; Haber et al. 2002; Toomre 2002).

Statistical properties of supergranulation flows have been investigated by the timedistance analysis of the surface gravity waves (f-modes). The results have revealed a vortex component due to the Coriolis effect, and, perhaps, a wave component of supergranulation, which may explain the long-standing puzzle why the supergranulation pattern rotates faster than the surface plasma (Duvall \& Gizon 2000; Gizon et al. 2002).

\subsection{Theory and Modelling}

Wave-theoretical methods for time-distance helioseismology have been formulated. They include effects of the finite wavelength, wave damping, and non-uniform distribution of the wave sources, and will lead to more accurate diagnostics of subsurface structures, particularly, at smaller scales (Birch \& Kosovichev 2000; Jensen et al. 2000; Birch et al. 2001; Gizon \& Birch 2002).

The recently developed method of dense-packed ring diagrams has allowed to make very efficiently synoptic maps of subsurface flows thus enabling the SSW studies (Haber et al. 2000).

A phase-sensitive formulation has significantly increased the capabilities of acoustic holography. It allows to measure small phase changes between the ingression and egression signals, caused by subsurface inhomogeneities and flows. However, inversion procedures for this method are not yet developed (Lindsey \& Braun 2000a).

The first attempt to develop a wave-form tomography for the Sun has been made, and the first results for supergranulation are very encouraging. This method has the potential of extracting more complete information about the subsurface structures and flows compared to the other methods, which use only specific characteristics of the wave form (travel times, phases etc) (Woodard 2002).

\section{References}

Basu, S., \& Antia, H.M. 2000, Solar Phys. 192, 469.

Birch, A.C., Kosovichev, A.G. 2000, Solar Phys. 192, 193.

Birch, A.C., et al. 2001, ApJ 561, L229.

Braun, D.C., \& Lindsey, C., 2001, ApJ 560, L189.

Chou, D.-Y. 2000, Solar Phys. 192, 241.

Chou, D.-Y., \& Dai, D.-C. 2001, ApJ 559, L175.

Duvall T.L. \& Gizon L. 2000 Solar Phys. 192, 177.

Duvall, T.L., Jr., \& Kosovichev, A.G., 2001, in: Recent Insights into the Physics of the Sun and Heliosphere:Highlights from SOHO and Other Space Missions, Proc. IAU Symp. 203, P. Brekke, B. Fleck, J.B. Gurman (eds.), ASP Conf. Ser., 159.

Giles, P.M. 1999, Time-Distance Measurements of Large-Scale Flows in the Solar Convection Zone, $\mathrm{PhD}$ Thesis, Stanford U.

Gizon, L., et al. 2000, JA\&A 21, 339.

Gizon, L., et al. 2001, in: Solar Encounter: The First Solar Orbiter Workshop, ESA SP-493, ESTEC, Noordwijk, 227.

Gizon, L., et al. 2002, Nature, submitted.

Gizon, L., \& Birch, A. C. 2002, ApJ, 571, 966.

Haber, D.A., et al. 2000, Solar Phys. 192, 335.

Haber, D.A., et al. 2002, ApJ 570, 855.

Hernandez, I.G., et al. 2000, ApJ 535, 454. 
Hurlburt, N.E., \& Rucklidge, A.M., 2000, MNRAS 314, 793.

Jensen, J.M., et al., 2000, Solar Phys. 192, 231.

Jensen, J.M., et al., 2001, ApJ 555, L193.

Kosovichev, A.G., et al. 2000, Solar Phys. 192, 159.

Kosovichev, A.G., et al. 2002a, Adv. Space Res. v.29.

Kosovichev, A.G., et al. 2002b, in: Magnetic Coupling of the Solar Atmosphere, ESA SP-505, ESTEC, Noordwijk.

Lindsey, C., \& Braun, D.C., 2000a, Solar Phys. 192, 261.

Lindsey, C., \& Braun, D.C., 2000b, Science 287, 1799.

Toomre, J. 2002, Science 296, 64.

Woodard, M.F. 2002, ApJ 565, 634.

Zhao, J., et al. 2001, ApJ 557, 384.

\section{Progress in the Study of the Solar Corona}

\section{J. Mariska}

Observational studies of the solar corona in the last three years have enjoyed an abundance of new high spatial resolution data from the Transition Region and Coronal Explorer (TRACE) mission (Handy et al. 1999). These narrow-band images, coupled with the continued analysis of observations from instruments on the Solar and Heliospheric Observatory (SOHO) and Yohkoh, have produced a wealth of new insights into the physics of the corona. Much of this research has focussed on understanding coronal loops, especially in active regions.

Examinations of these new observations have revealed that the observed characteristics of many loops in active regions disagree with the simple scaling laws derived using earlier observations (Aschwanden, Nightingale \& Alexander 2000; Lenz et al. 1999a, 1999b). Instead of the loops displaying a steep temperature increase through the transition region followed by a small, but measurable, rise from the top of the transition region to the loop apex, the coronal portions of the loops appear to be more uniform with a temperature of about $1 \mathrm{MK}$. Moreover, the intensity distribution suggests that the density is falling more slowly with increasing height than would be expected in hydrostatic equilibrium (Aschwanden, Schrijver \& Alexander 2001).

These new observations have led to continued speculation about the nature and location of coronal heating. Heating at loop footpoints will produce some of the characteristics seen in the TRACE data (Aschwanden, Schrijver \& Alexander 2001). But it now appears that the best explanation for the observations will involve time-varying substructure, perhaps similar to that discussed by Klimchuk \& Cargill (2001). This dynamic picture is further supported by observational evidence from SUMER spectra of flows in active region loops (Winebarger et al. 2002).

TRACE observations have also revealed so-called EUV moss in active regions (Handy et al. 1999; Berger et al. 1999). These structures appear to be the transition region footpoints of very hot coronal loops. Models for the loops that might be anchored in the moss suggest that they have peak temperatures of 3-10 MK and filling factors of about 0.1 (Martens et al. 2000).

Higher in the corona, detailed analysis of observations from the Ultraviolet Coronal Spectrometer on SOHO have resulted in new models for coronal holes (Cranmer et al. 1999), and insights into the role of ion cyclotron resonance heating in these sources of high-speed solar wind (Cranmer, Field \& Kohl 1999). Detailed line intensity profile measurements using the SOHO SUMER experiment have provided additional constraints on the physical conditions low in the corona in both coronal holes and the quiet Sun (e.g., Doschek et al. 2001; Feldman et al. 1999). 


\section{References}

Aschwanden, M.J., Schrijver, C.J., \& Alexander, D. 2001, ApJ, 550, 1036.

Aschwanden, M.J., Nightingale, R.W., \& Alexander, D. 2000, ApJ, 541, 1059.

Berger, T.E., De Pontieu, B., Schrijver, C.J., \& Title, A.M. 1999, ApJ, 519, L97.

Cranmer, S.R. et al. 1999, ApJ, 511, 481.

Cranmer, S.R., Field, G.B., \& Kohl, J.L. 1999, ApJ, 518, 937.

Doschek, G.A., Feldman, U., Laming, J.M., Schühle, U., \& Wilhelm, K. 2001, ApJ, 546, 559.

Feldman, U., Doschek, G.A., Schühle, U., \& Wilhelm, K. 1999, ApJ, 518, 500.

Handy, B.N. et al. 1999, Solar Phys., 187, 229.

Klimchuk, J.A., \& Cargill, P.J. 2001, ApJ, 553, 440.

Lenz, D.D., Deluca, E.E., Golub, L., Rosner, R., \& Bookbinder, J. A. 1999a, ApJ, 517, L155.

Lenz, D.D., Deluca, E.E., Golub, L., Rosner, R., Bookbinder, J. A., Litwin, C., Reale, F., \& Peres, G. 1999b, Solar Phys., 190, 131.

Martens, P.C.H., Kankelborg, C.C., \& Berger, T. E. 2000, ApJ, 537, 471.

Winebarger, A.R., Warren, H., van Ballegooijen, A., DeLuca, E.E., \& Golub, L. 2002, ApJ, $567, \mathrm{~L} 89$

\section{Solar Instrumentation}

\section{P. Venkatakrishnan}

The end of the previous century witnessed great strides in solar instrumentation as evidenced by space based instruments like YOHKOH, SOHO and TRACE and ground based facilities like THEMIS. This impressive record seems to continue into the new millennium as will be seen in the following report based on the reports presented in journals and meetings.

\subsection{Space Instrumentation}

One of the major accomplishments in this period has been the launch of RHESSI, which is a Small Explorer mission selected by NASA to investigate the physics of particle acceleration and energy release in solar flares (Lin \& Dennis 2001). RHESSI utilizes Fourier - Transform imaging with 9 bi-grid rotating modulating collimators and cooled germanium detectors to make spectra and images in X-rays and Gamma rays from $3 \mathrm{keV}$ to $17 \mathrm{MeV}$. A Solar Xray Spectrometer (SOXS) to obtain total sunlight spectra in a similar range (Umapathy et al. 2001) is slated to be launched in 2002 on an Indian geostationary satellite GSAT-2. NOAA's GOES-12 weather satellite, launched into geosynchronous orbit on July 232001 , carried NOAA's first Solar X-ray Imager (SXI) which provides images with a 1 minute cadence, 10 arcsec spatial resolution and in a wavelength range of 0.6 to $6.0 \mathrm{~nm}$ (Hill et al. 2001). The Very-high-resolution Advanced Ultraviolet Telescope (VAULT) experiment consisting of a $30 \mathrm{~cm}$ UV telescope followed by a spectroheliograph was successfully launched on May 71999 on a Black Brant sounding rocket vehicle and obtained images of the upper chromosphere with a resolution of 0.33 arcsec (Korendyke et al. 2001). A prototype hard $\mathrm{X}$-ray spectrometer, developed jointly by the Astronomical Institute of the Czech Republic and the Space Environment Center of NOAA is presently in polar orbit aboard the MTI satellite to validate a methodology based on progressive spectral hardening for prediction of proton flares (Farnik et al. 2001). A more application oriented experiment called the Solar EUV Experiment (SEE) on NASA's TIMED spacecraft, measures the irradiance of the highly variable solar EUV radiation in the wavelength range of $0.1 \mathrm{~nm}$ to $195 \mathrm{~nm}$ (Woods et al. 2002). 


\subsection{Balloon Experiments}

In January 2000 , an $89 \mathrm{~cm}$ f $/ 1.5$ Ritchey-Chrétien solar telescope flew for 18 days suspended from a balloon in the stratosphere above Antarctica and obtained sharp and stable images of small scale solar features (Bernasconi et al. 2000). A hard X-ray spectrometer system, to be flown on a balloon, was developed using an array of $16 \mathrm{CdTe}$ detectors each of $10 \mathrm{~mm}$ $\times 10 \mathrm{~mm} \times 0.5 \mathrm{~mm}$ in size. The goal is to achieve a $3 \mathrm{keV}$ energy resolution over an energy range of $15-100 \mathrm{keV}$ for observing non-thermal and thermal components of the solar flare hard X-ray emission (Kobayashi et al. 2001).

\subsection{Ground Based Instruments}

R.H. Hammerschlag's Dutch Open Telescope (DOT) project progressed very well, in particular with the respect to the speckle reconstruction started at the DOT by Sütterlin (Sütterlin 2001). In the meantime, Hammerschlag and Bettonvil have designed multiwavelength optics to feed the multi-camera system with different beams including $\mathrm{CaII} \mathrm{K}$ and $\mathrm{H}_{\alpha}$. The first synchronous two-channel data were obtained in October 2001 (Rutten 2002). The New Swedish Vacuum Telescope with a 1-m aperture singlet lens and a Schupmann corrector saw first light in March 2002 (Anonymous 2002). An innovative approach of using the combined beams of three 30-cm telescopes was successfully tested at the Observatoire du Paris, Meudon (Damé 2002). SOLIS (Synoptic Optical Long-term Investigations of the Sun) is a project to replace several of NSO's existing synoptic instruments with a modern, state-of-the-art observing system (Harvey et al. 2000; Keller 2000). It consists of the Integrated Sunlight Spectrometer (ISS) and a $50 \mathrm{~cm}$ aperture Vector Spectromagnetograph (VSM) to produce full disk vector magnetograms which will be supplied to the community. Magneto Optical Filters have been used to develop narrow band filtergraphs for the VAMOS project (Severino et al. 2001) and for Doppler measurements using the $\mathrm{K} 769.9 \mathrm{~nm}$ line at the Prairie View Solar Observatory (Huang \& Pojoga 2002). The traditional Lyot filter was used for the new digital $\mathrm{H}_{\alpha}$ observations at the Bohyansan Optical Astronomical Observatory in Korea (Lee et al. 2001). It might be of interest to some observers that the Nanjing Astronomical Instrument Center of the Chinese Academy of Sciences (Li et al. 2001) can make and repair Lyot filters. Portable instruments required for total solar eclipse observations, like the fast imaging system to detect coronal oscillations (Phillips et al. 2000), the polarimeter of the Udaipur solar Observatory (Ambastha et al. 2002) and the Fabry-Perot etalon based interferometric imaging system of the Physical Research Laboratory (Chandrasekhar \& Ashok 2002) were successfully deployed.

The second generation of the Zurich Imaging Polarimeter, ZIMPOL II, allows us to record images of the full Stokes vector (all four Stokes parameters) with unprecedented polarimetric sensitivity (Povel 2001). The system has been successfully used at a number of different telescopes (Stenflo et al. 2002). A new, UV-sensitive version of ZIMPOL II has been developed during the last two years which is sensitive down to about $300 \mathrm{~nm}$, beyond the atmospheric cutoff. The new system was successfully applied for scientific programs. Spectrograph based polarimetry was employed at the $25 \mathrm{~cm}$ coronagraph of the Norikura Solar Observatory (Shimoda et al. 2001) and at the Coudé spectrograph of the Big Bear Solar Observatory (Zirin \& Cameron 2001). The telescope polarization of the 3 mirror coelostat system of the Kodaikanal Observatory was successfully eliminated using ellipsometry and the corrected Stokes profiles were used to measure the vector magnetic field of active regions (Sankarasubramanian et al. 2000). A program of measuring large scale solar magnetic fields using the Stokes I and V profiles has been initiated at the Sayan Solar Observatory (Demidov et al. 2002). New backend instruments were also developed for radio telescopes like a broad band spectrometer operating at $4.5 \mathrm{GHz}$ to $7.5 \mathrm{GHz}$ at the Purple Mountain Observatory (Xu et al. 2001) and a digital spectrograph for the Gauribidanur radioheliograph operating at $30 \mathrm{MHz}$ to $80 \mathrm{MHz}$ (Ebezener et al. 2001). 


\subsection{Upgrades}

The GONG program has been successfully upgraded at all the 6 sites with the replacing of the $256^{2}$ pixel camera by a $1024^{2}$ pixel camera. Associated readout and data archiving upgrades were also implemented (Leibacher 2001). The Marshall Space Flight Center's solar group has announced the successful upgrade of their tower magnetograph resulting in an increase of spatial resolution from $2.5 \mathrm{arcsec} / \mathrm{pixel}$ to $0.64 \mathrm{arcsec} / \mathrm{pixel}$ (Adams et al. 2001). The Mount Wilson Synoptic Magnetic field program has installed a new 24channel magnetograph (Ulrich et al. 2002). The Big Bear Solar Observatory upgraded its digital vector magnetograph (DVMG) with an easy to use GUI observing tool (Spirock et al. 2001) to integrate the data with the RHESSI synoptic archive. The Owens Valley Solar Array (OVSA) underwent extensive hardware and software upgrades. In preparation for the launch of RHESSI (Gary et al. 2000).

\section{References}

Adams, M., Hagyard, M.J., West, E.A., \& Smith, J.E. 2001, AGU Spring Meeting.

Ambastha, A., Gosain, S., \& Venkatakrishnan, P. 2002, in: Probing The Sun With High Resolution, Proc. Silver Jubilee Meeting of Udaipur Solar Observatory, Narosa, S.C. Tripathy, P. Venkatakrishnan (eds.), submitted.

Anonymous, 2002 , http://www.astro.su.se/groups/solar/first-light.html

Bernasconi, P.N., Rust, D.M., Eaton, H.A., \& Murphy, G.A. 2000, AAS SPD Meeting.

Chandrasekhar, T. \& Ashok, N.M. 2002, in: Probing The Sun With High Resolution, Proc. Silver Jubilee Meeting of Udaipur Solar Observatory, Narosa, S.C. Tripathy, P. Venkatakrishnan (eds.), submitted.

Damé, L., 2002, in: Probing The Sun With High Resolution, Proc. Silver Jubilee Meeting of Udaipur Solar Observatory, Narosa, S.C. Tripathy, P. Venkatakrishnan (eds.), submitted.

Demidov, V.S., Zhigalov, V.V., Pescherov, V.S., \& Grigorev, V.M. 2002, Solar Phys., in press.

Ebenezer, E., Ramesh, R., Subramanian, K.R., Suderarajan, M.S., \& Sastr, Ch. V. 2001, A\&A, 367, 1112.

Farnik, F., Garcia, H., \& Karlicky, M. 2001, Solar Phys., 201, 357.

Gary, D.E., Hurford, G.J., Lee, J., \& Gallagher, P.T. 2000, AAS SPD Meeting.

Harvey, J., \& SOLIS team, 2000, AAS SPD Meeting.

Hill, S.M., Pizzo, V.J., \& Baleh, C.C. 2001, AGU Fall Meeting.

Huang, T.-S., \& Pojoga, S. 2002, AAS Meeting 200.

Keller, C.U. 2000, JA\&A, 21, 127.

Kobayashi, K., Tsuneta, S., Tamura, T., Kumazai, K., Katsukawa, Y., Kobo, S., \& Yamagami, T. 2001, in: Proc. YOHKOH 10th Anniversary meeting, P.C.H. Martens, D. Cauffman (eds.), COSPAR Colloquia Series, Elsevier Science.

Korendyke, C.M., Vourlidas, A., Cook, J., Dere, K.P., Howard, R.A., Morrill, I.S., Moses, J.D., Moulton, N.K., \& Socker, D.G. 2001, Solar Phys., 200, 63.

Lee, C.-W., Moon, YY.-J., Park, D., Jang, R.-H., \& Kim, K.-S. 2001, J. Korean Astron. Soc., 34, 111.

Leibacher, J. 2001, AAS Meeting 198.

Li, T., Mao, W., Hai, L., \& Zhu, Y. 2001 Progress in Astron., 19, 331.

Lin, R.P., \& Dennis, B. 2001, AGU Spring Meeting.

Phillips, K.J.H., Read, P.D., Gallagher, P.T., Keenan, F.P., Rudavy, P., Rompolt, B., Berlicki, A., Buzylko, A., Diego, F., Barneley, R., Smartt, R.N., Pasachoff, J.H., \& Babcock, B.A. 2000, Solar Phys., 193, 259. 
Povel, H. 2001, in: Magnetic Fields Across the Hertzsprung-Russel Diagram, G. Mathys, S.K. Solanki, D.T. Wickramasinghe (eds.), ASP Conf. Ser., 248, 543.

Rutten, R. 2002 in: Probing The Sun With High Resolution, Proc. Silver Jubilee Meeting of Udaipur Solar Observatory, Narosa, S.C. Tripathy, P. Venkatakrishnan (eds.), submitted.

Sankarasubramanian, K., Srinivasulu, G., Ananth, A.V., \& Venkatakrishnan, P. 2000, JA\&A, 21, 241.

Severino, G., Moretti, P.F., Oliviero, M., \& VAMOS team 2001, in: Proc. SOHO 10/GONG 2000 Workshop, A. Wilson (ed.), ESA SP-464, ESTEC, Noordwijk.

Shimoda, K., Ichimoto, K., Fukuda, T. \& Shio, J. 2001, Report of the National Astronomical Observatory of Japan, 5, 97.

Spirock, T.J., Denker, C., Varshik, J., Shumko, S., Qia, J., Gallagher, P., Chae, J., Goode, P., \& Wang, H. 2001, AGU Spring Meeting.

Stenflo, J.O., Gandorfer, A., Holzreuter, R., Gizler, D., Keller, C.U., \& Bianda, M. 2002, A\&A, 389, 314 .

Sütterlin, P. 2001, A\&A, 374, L21.

Ulrich, R. K., Evans, S., Boyden, J.E., \& Webster, L. 2002, ApJS, 139, 259.

Umapathy, S.N., Sharma, M.R., Solanky, C., Kulkarni, R., Shah, A.B., Vadher, N.M., Jain, R., Jain, A.K., \& Sreekumar, P. 2001, Bull. Astron. Soc. India, 29, 475.

Woods, T.N., Eparvier, F.G., Woodraska, D., Rottman, G.J., Solomon, S.C., Roble, R., de Toma, G., Lean, J., Tobiska, W.K., \& Bailey, S.M. 2002, AGU Spring Meeting.

Xu, F.-Y., Yao, Q.-J., Meng, X., \& Wu, H.-A., 2001, Chinese J. Astron. Astrophys., 1, 469.

Zirin, H., \& Cameron, R. 2001, AGU Spring Meeting.

\section{Solar Surface Magnetism}

\section{J. Wang}

\subsection{Magnetic Field Measurements}

In the period reported upon here, a number of new diagnostics of the magnetic field have been demonstrated to work and older diagnostics have been carefully investigated. Thus, the molecular Zeeman effect, which can be applied to the studies of solar and stellar magnetism, has been explored. The first spectral synthesis of Zeeman-split Stokes I and V profiles of $\mathrm{OH}$ lines, and the $\mathrm{MgH}$ green system and TiO $\gamma$-system were presented (Berdyugina \& Solanki 2001; Berdyugina et al. 2000). Infrared lines as probes of solar magnetic features have been extensively explored (see Rüedi et al. 1999). Using a very sensitive infrared spectropolarimeter, Lin, Penn \& Tomczyk (2000) succeeded in measuring the weak Stokes $\mathrm{V}$ circular polarization profiles of the strong coronal emission line, Fe XIII 10747, above active regions.

Hagyard et al. (2000), Zhang (2000) and Bao et al. (2000) evaluated the effects of Faraday rotation on the observed transverse magnetic fields by comparing different types of vector magnetic field measurements. They brought up the conclusion that Faraday rotation could be a significant problem if the measurements were made at the line center.

Time sequence of the quiet-sun magnetic fields at a resolution of 0.2 arcsec by combining the adaptive optics system at the Dunn Solar Telescope with the Zurich Imaging Polarimeter I (ZIMPOL) and processing the data with Phase-Diverse Speckle Imaging and speckle deconvolution was obtained by Keller et al. (2000, American Astronomical Society, SPD meeting $32,03.01$ ). 


\subsection{Activity-Associated Magnetic Changes}

For the Bastille Day flare/CME on July 14, 2000, Zhang et al. (2001) identified that the only obvious magnetic changes were the flux cancellation at several key sites of the long filament channel. This is the first report on the association of global CME and smallscale flux cancellation. The same irreversible variation was independently found from MDI magnetograms (Kosovichev \& Zharkova 2001). Deng et al. (2001) suggested a magnetic energy transport from the lower atmosphere to the corona in the event, evidenced by the creation of coronal flux ropes (Yan et al. 2001).

It becomes clear that there are two types of magnetic changes: irreversible and impulsive. The former includes flux emergence, cancellation, evolving magnetic shear and helicity ( $\mathrm{Li}$ et al. 2000; Asai et al. 2001; Mathew \& Ambastha 2000; Kim et al. 2001; Kurokawa et al. 2002; Spirock et al. 2002); the latter is probably caused by high-energy particles bombarding the solar surface (Lozitsky et al. 2000; Kosovichev \& Zharkova 2001). Falconer (2001) suggested ways of CME prediction based on the irreversible changes in the magnetic vector.

Magnetic reconnection in the photosphere and lower atmosphere has also attracted recent attention (Tarbell et al. 2000; Chen et al. 2001; Ji et al. 2001). High-cadence and high-resolution flare observations indicated that some impulsive compact flares involve low-lying magnetic loops or magnetic reconnection at small altitudes (Wang \& Qiu 2002).

\subsection{Topological Structures and Quantities}

Concepts of separatrix, separator, quasi-separatrix layer, and Bald-patch were found to be relevant not only to flares, but also to coronal heating (Wang et al. 2000). The work on 3D modeling of complex fields created wholly new terms, such as fan and spine reconnection (Priest \& Forbes 2002). A more general term, magnetic interface, was used to describe the interaction of topology-independent flux systems (Zhang \& Wang 2002).

Magnetic helicity has turned out to be extremely important in constraining the energy release. DeVore (2000) first quantitatively investigated the generation of magnetic helicity by solar differential rotation acting on emerged bipolar sources of flux. Chae et al. (2001) suggested a way of observationally determining helicity transport. Démoulin et al. (2002a) concluded that the main source of helicity was the inherent twist of the magnetic flux tubes forming the active region. Démoulin et al. (2002b) further deduced that the efficiency of shear motion in creating the magnetic helicity depended on both magnetic configuration and shear properties.

\subsection{Magnetic Carpet}

Flux emergence, cancellation, coalescence, and fragmentation produce the evolution of the so-called 'magnetic carpet' of the quiet photosphere (Parnell 2001). Hagenaar (2001) estimated that the total amount of flux emerging in ephemeral regions was $5 \times 10^{23} M x d^{-1}$, while Title (2000) gave an estimate of $7 \times 10^{22} M x d^{-1}$, and Chae et al. (2001) found $3 \times 10^{23} M x d^{-1}$. Worden \& Harvey (2000) estimated that approximate $1.1 \times 10^{23} M x d^{-1}$ new background magnetic flux was needed to sustain the quiet sun magnetic field.

However, flux emergence also takes place at even smaller scales. De Pontieu (2002) presented observations of flux emergence in the internetwork. Socas-Navarro \& Sánchez Almeida (2002) suggested a magnetic field with complicated topology and mixed polarities within the resolution element based on novel inversions of Stokes profiles. The inexhaustible interaction among intranetwork, network, and ephemeral regions was found to be the origin of mini-filament eruption, microflare and macrospicule (Wang et al. 2000). This interaction might provide enough energy for coronal heating. 


\subsection{Global Pattern and Physics}

Solanki, Schüssler \& Fligge (2002) modelled the time evolution of both the open source flux and the total (cycle-related) magnetic flux at the solar surface since 1700, and indicated that the total surface flux has doubled in the first half of the last century. The modelled open flux is in good agreement with that reconstructed by Lockwood, Stamper \& Wild (1999). Makarov et al. (2002) suggested that this behavior might be explained by the increase of the area of polar caps.

The 3-D MHD numerical simulation shed new lights on understanding the elementary physical processes involved. The simulated dynamic emergence of a twisted, $\Omega$-shaped tube, from the top layer of the solar convection zone into the atmosphere, reproduced all the major observational features of active region development (Fan 2001). Successful efforts were made by Schrijver and his co-workers to uniformly understand the physics of stellar magnetic activity based on the empirical solar knowledges (see Schrijver 2001).

\section{References}

Asai, A., Ishii, T.T., \& Kurokawa, H. 2001, ApJ, 555, L65.

Bao, S.D., Pevtsov, A.A., Wang, T.J. \& Zhang, H.Q. 2000, Solar Phys., 195, 75.

Berdyugina, S.V. et al. 2000, A\&A, 364, L101.

Berdyugina, S.V., Solanki, S.K. 2001, A\&A, 380, L5.

Chae, J., Martin, S.F., Yun, H.S., Kim, J., Lee, S., Goode, P.R., Spirock, T., \& Wang, H. 2001, ApJ, 548, 497.

Chen, P.F., Fang, C., \& Ding, M.D., 2001, Chinese J. A\&A, 1, 176.

Démoulin, P., Mandrini, C.H., Van Driel-Gesztelyi, L., Lopez Fuentes, M.C., \& Aulanier, G. 2002a, Solar Phys., 207, 87.

Démoulin, P., Mandrini, C.H., Van Driel-Gesztelyi, L., Thompson, B.J., Plunkett, S., Kovári, Zs., Aulanier, G., \& Young, A. 2002b, A\&A, 382, 650.

Deng, Y., Wang, J., Yan, Y., \& Zhang, J. 2001, Solar Phys. 204, 11.

De Pontieu, B. 2002, ApJ 569, 474

De Vore, C.R. 2000, ApJ, 539, 944.

Falconer, D.A., 2001, JGR, A11, 25185.

Fan, Y., 2001, ApJ, 554, L111.

Hagenaar, H.J. 2001, ApJ, 555, 448.

Hagyard, M.J., Adams, M.L., Smith, J.E., \& West, E.A. 2000, Solar Phys., 191, 309.

Ji, H.S., Song, M.T., \& Huang, G.L. 2001b, ApJ, 548, 1087.

Keller, C.U., Rimmele, T.R., Paxman, R.G., Seldin, J.H., Carrara, D., \& Gleichman, K. 2000, AAS SPD Meeting 32.

Kim, J.-H., Yun, H.S., Lee, S., Chae, J., Goode, P.R., \& Wang, H. 2001, ApJ, 547, L85.

Kosovichev, A.G. \& Zharkova, V.V. 2001, ApJ, 550, L105.

Kurokawa, H., Wang, T., \& Ishii, T.T., 2002, ApJ, 572, 598.

Li, H. et al. 2000, PASJ, 52, 465; 52, 483.

Lin, H., Penn, M.J., \& Tomczyk, S. 2000, ApJ, 541, L83.

Lockwood, M., Stamper, R., \& Wild, M.N. 1999, Nature, 399, 437.

Lozitsky, V.G., Baranovsky, E.A., Lozitska, N.I., \& Leiko, U.M. 2000, Solar Phys., 191, 171.

Makarov, V.I., Tlatov, A.G., Callebaut, D.K., \& Obridko, V.N. 2002, Solar Phys., 206, 383.

Mathew, S.K., \& Ambastha, A. 2000, Solar Phys., 197, 75.

Parnell, C.E. 2001, Solar Phys., 200, 23.

Priest, E. R. \& Forbes, T. G., 2002, Solar Phys., 205, 249. 
Rüedi, I., Solanki, S.K., \& Keller, C.U., 1999, A\&A, 348, L37.

Schrijver, C.J. 2001, ApJ, 547, 475.

Socas-Navarro, H., \& Sánchez Almeida, J. 2002, ApJ, 565, 1323.

Solanki, S. K., Schüssler, M., \& Fligge, M. 2002, A\&A, 383, 706.

Spirock, T.J., Yurchyshyn, V.B., \& Wang, H., 2002, ApJ, 572, 1092.

Tarbell, T. D., Ryutova, M., \& Shine, R., 2000, Solar Phys., 193, 195.

Title, A.M. 2000, Philos. Trans. R. Soc. London A, 358, 657.

Wang, H. \& Qiu, J. 2002, ApJ, 568, 408.

Wang, J., Li, W., Denker, C., Lee, C., Wang, H., Goode, P.R., McAllister, A., \& Martin, S.F. 2000, ApJ, 530, 1071.

Worden, J. \& Harvey, J. 2000, Solar Phys., 195, 247.

Yan, Y., Aschwanden, M.J., Wang, S., \& Deng, Y. 2001, Solar Phys., 204, 27.

Zhang, H. 2000, Solar Phys., 197, 235.

Zhang, C. \& Wang, J. 2002, Solar Phys., 205, 303.

Zhang, J. et al. 2001, ApJ, 548, L99. 